\title{
Platform Establishment: Navigating Competing Concerns in Emerging Ecosystems
}

\author{
Hosea A. Ofe \\ Umeå University, Sweden \\ hosea.ofe@informatik.umu.se
}

\author{
Johan Sandberg \\ Umeå University, Sweden \\ jsberg@informatik.umu.se
}

\begin{abstract}
Digital Platforms impose organizing logics on ecosystems. Dependent on their configuration, they enable certain practices, relationships, and value distribution among actors while preventing alternatives. Incumbent platforms often have a strong power to implement contested configurations since they control access to attractive user groups/markets. However, emerging platforms have a small degree of bargaining power in relation to key actors since they have not yet achieved such a position. Although numerous studies detail governance strategies for incumbent platform ecosystems, research on how platform providers navigate competing concerns in emerging platform ecosystems remain rare. We report on a study of the establishment and continuous dynamics of a digital platform used for service innovation. We inductively identify a pattern of the dynamics in this navigation process, locate four salient tensions driving these dynamics, and provide insights on how the platform provider navigated them.
\end{abstract}

\section{Introduction}

Digital platforms, i.e. the "software-based products or services that serves as a foundation upon which outside parties can build complementary products or services" [1:5] provide organizations great opportunities for value creation. However, we are only starting to grasp the processes through which digital platforms emerge and establish a significant user base [2]. The literature on platform governance has largely focused on strategies to stimulate the development of services, coordinate and monetize the activities of distributed and heterogeneous actors participating in established digital platform ecosystems [3-6]. A salient theme in this body of research is the tensions resulting from the interactions of competing actors and platform owners in relation to different concerns. Examples include tensions related to identity [7], standard- variety, control-autonomy and collective-individual [4]. For the provider, a key strength of the platform as an organizing logic is the ability to unite and coordinate agents in the ecosystem towards its strategic goals [8]. These agents may have distinct understandings of the current situation, conflicting goals, and incompatible ideas of how to advance the ecosystem, which we here refer to as competing concerns. Most studies examining platform governance strategies and the effects on the wider ecosystem have tended to focus on how platform providers address competing concerns in established platform ecosystems $[6,9]$. Thus, extant research details the strategies of incumbents such as Apple, Google, or Intel in leveraging their market position and strategic control over key resources to influence an ecosystem. Such studies build on the premise that, as an owner of a central enabling bottleneck, the platform owner's power and bargaining position often greatly exceeds that of independent suppliers of complementary components [10]. Platform owners will often have "bouncer's rights" [11] to grant or deny access to the system and stipulate conditions associated with admittance. These empirical settings have provided insights into governance of third-party developers in platform ecosystems and choices regarding a platform's economic scope and scale [10].

However, establishing an ecosystem is significantly different from reinforcing an incumbent ecosystem. Unlike an incumbent platform ecosystem with a relatively stable value proposition and user base, an emerging platform's value can be uncertain and obscured since it is often tightly connected to the user base and interactions in the ecosystem. Emerging platforms, with unstable user bases, will often have relatively small bargaining power or strategic control. i.e. the influence exerted on other members [12] since the value of the "bouncer's rights" is dependent on the size and attractiveness of the entire ecosystem. Although incumbent digital platforms do not retain full control over design decisions [5], emergent platforms are likely to meet distinct challenges when enforcing change and managing competing concerns. The 
emphasis on mature and industry-leading platform ecosystems has led to a lack of knowledge of the processes and mechanisms through which platform providers deal with competing concerns during platform establishment. An increased understanding of governance dynamics in emerging platform ecosystems can help provide insights into the design of digital platforms [2]. Against this backdrop, this research explores how competing concerns shape the trajectory of nascent digital platform ecosystems.

To this end, we report on a case study of a platform, Trafiklab, an initiative aimed at fostering an ecosystem of data providers and developers engage in developing digital services based on public transport data. Our analysis reveals multiple cycles of recurrent challenges to the status quo and associated responses. We provide a process model of the dynamics of platform establishment where new sociotechnical configurations arise from, and give rise to, tensions related to competing concerns. We provide insights into the emergent nature of this process.

\section{Digital Platform Establishment}

Digital platforms provide infrastructures for interactions between disparate groups, enable re-use of core components that provide functionality for peripheral modules, and coordinate the associated ecosystem through sociotechnical resources such as pricing, rules and boundary resources [3, 5, 13, 14]. Since they impose organizing logics, platforms can broadly be understood as meta-organizations that coordinate agents in a wider system [8]. The design of sociotechnical resources is the main vehicle through which a platform provider influences its ecosystem i.e. third-party applications and complementors participating to create value based on a platform's interfaces [1]. Through the design of technological and social resources, a platform provider can specify decision rights, control how the platform is used to create services, and structure incentives to encourage or discourage certain actions by users in the ecosystem [1].

A platform's life cycle consists of three phases: (1) emergence of an ideal solution, (2) progression along a technology maturity curve, and (3) its uptake by group of prospective end-users [1]. Although the emergence phase is inherently fragile and can define the fate of a platform, few studies explore platform strategies and their dynamics in this phase. Strategy-oriented literature on platforms generally high-light pricing policies, such as subsidies and premium fees, to kickstart, or "ignite", a platform [14, 15]. By subsidizing prospective users, a new platform entrant can draw on initial users to attract others [15]. This perspective builds on the premise that once the platform "ignites" by attaining a critical mass of users, it becomes selfsustaining [16]. Literature has also explored nonpricing incentives such as timely notifications of changes affecting modules (e.g.- apps) as essential in attracting users and increasing the quality of external applications [17]. Developers may multi-home across multiple platforms, the capability to search for ways to distribute and monetize services in the ecosystem is an essential aspect in enriching a platform's innovation habitat [18]. Degrees of openness and control is another widely acknowledged design consideration whereby deliberatively and progressively relinquishing control, a platform owner allows external actors to build complementary components that extend the platform ecosystem [10]. Labelled as coring, a set of technological and business functions entrants need to grow an ecosystem has been suggested [19]. Examples of these functions include ensuring that the platform serves a relevant function for an industry, ensuring dependence between components and protecting its core source of revenue [19]. Although the notion of coring includes a high-level description of what functionality new platform providers need to offer, how such functions are operationalized when establishing digital platforms has received limited attention.

\subsection{Competing Concerns and Digital Platform Ecosystem Governance}

Establishing a new platform involves reconfiguring organizing logics and relationships across multiple organizations and actors in an ecosystem. A new platform carries distinct structures for how to arrange and conduct exchanges as technical elements are coupled and new types of interactions among agents are facilitated. As such, it brings together multiple, distributed actors with different perspectives that need to be reconciled for the platform to become an accepted de facto standard. The structural configuration of digital platforms is, however, not static. Rather, it is subject to recurrent challenges from the distributed set of actors in the wider system of use $[5,20]$. This is partly due to the malleability of digital technology that "exhibits a procrastinated binding of form and function [21] meaning that new capabilities can be added after a product or tool has been designed and produced" [22:1399]. Thus, the existing arrangement of resources and power within ecosystems continues to evolve throughout the lifetime of a digital platform, leading to disputed designs, unintended consequences and tensions i.e. differing, conflicting and competing demands within, and across, 
stakeholders [23, 24]. A platform provides digital resources, such as APIs, that are shared among a large set of users. Control over their use is distributed across multiple stakeholders and contexts [20]. Fluidity of resources poses significant tensions for the platform providers. For example, tensions can arise from distinct perspectives on the standards and quality of service that can be generated across the ecosystems or negotiating an optimal level of control that the platform provider can institute to provide flexibility to coordinate autonomous actors across the ecosystem [4]. Established platforms are often able to exercise significant degrees of control to increase predictability of individual behavior and ecosystem trajectories. Based on the interactions among their accumulated user bases, they can generate an understanding of the nature of these interactions and governance mechanisms and draw on methods such as pattern recognition (e.g., Netflix can recommend content based on similar users). Moreover, a platform's potential merit is largely emergent, arising in-vivo since it is tightly connected to the user base and interactions in the ecosystem. Understanding the types of interactions and value that the platform offers to users is challenging for emerging platforms with relatively small user bases. Not least since prospective platform users may generally postpone adoption or only partly commit until the value and participation of other users is realized [25].

Given that a platform's sociotechnical design is prone to evolve over time, and is subject to different re-combinations, platforms can draw on distinct degrees of architectural leverage in production, innovation, and transaction [26]. Production leverage describes how platforms enable reuse of core assets and, through interfaces, allow sharing of these with actors in the ecosystem, thereby enabling economies of scale and scope. For example, Apple and Google provide the architectural base of APIs that enable developers to create applications interoperable across wider ecosystems. Innovation leverage also refers to the sharing of resources, but for innovative purposes through recombination and specialization [27]. Innovation leverage arises from digital platforms' capacity to facilitate learning and creativity by converting search from distant to local [28]. The search for innovative solutions and knowledge resources is path dependent as satisfying actors, in general, will draw on previous connections, experiences, and knowledge i.e. explore options in the "local" domain. Such a search is more cost-efficient since it leverages the actor's current expertise, routines, cognitive frame and absorptive capacity, with the risk of better alternatives not being considered [29]. Distant search i.e. exploring options further away from the actor's own practices and knowledge entail greater search costs. Transaction leverage refers to the intermediary role platforms play in enabling transactions of various kinds by providing infrastructure for interactions and exchange, thereby increasing efficiency and reducing search costs. In digital platform ecosystems, these exchanges are naturally founded on information transactions. An example is how Airbnb facilitates transactions between users by reducing the search time required for lease and rental apartments.

As digital platforms provide resources that are dynamic and a function of human appraisal [30], they enable new actors across the ecosystems to engage in practices that promotes their interest as they seek to create value. As actors may hold different beliefs about the platform's potential value, e.g. due to information asymmetries [13], the platform provider is susceptible to different and contradictory responses as external actors gradually shape an understanding of valuecreating interactions through trial-and-error practices. During such learning processes, ecosystem actors are likely to discover and engage in practices that deviate from current practices, making tensions salient, and potentially contradictory by revealing plurality, scarcity or hitherto unrecognized changes [4]. Plurality in the context of digital ecosystems refers to the availability of similar options while scarcity arises from competition regarding specific resources. A high degree of change in emerging platform ecosystems, results in uncertainties regarding economic returns from investments and opportunistic behavior. Providers are therefore required continuously adapt to the needs of actors while dealing with uncertainty in the technological trajectories. For emerging platforms, due to their relatively smaller influence over resources across the ecosystem, these uncertainties can subject the platform provider to contradictory practices as they seek to alter and re-align themselves with multiple identities and practices of actors in the ecosystem [7].

To explore how tensions arising from competing concerns and shape the evolutionary trajectory, we conceptualize digital platforms as an evolving set of sociotechnical resources, carrying distinct architectural leverage, varying over time, and enabling different types of connections among constitutive modules and agents.

\section{Research Design}

We studied Trafiklab, a start-up in the public transport industry in Sweden, launched in September 2011 by a group of Public Transport Operators [PTOs] led by Samtrafiken. Trafiklab aims to serve as a hub for PTOs, providing open data and APIs to developers 
and public transport users. It has an overarching goal of facilitating the development of digital services based on public transport data. Between 2011-2017, approximately 3000 users registered, 2200 services and prototype applications were developed. To this end, Trafiklab was deemed a suitable study object since it is currently in the developing phases of the platform life cycle. Semi-structured interviews, internal reports, and weblogs were used as data sources. In total, we conducted 48 interviews and used 29 internal documents and blog posts. Interviews were conducted between 2015-2018. The overall duration of the interviews was 36 hours, with an average interview time of 1 hour 5 minutes. As we were interested in exploring concerns and actions dating from Trafiklab's inception, interviewees selected needed to have knowledge regarding Trafiklab's inception and to have been involved at decision-making levels that affected Trafiklab's trajectory.

\subsection{Data Analysis}

Our data analysis was guided by an inductive approach, following an initial coding phase, focused coding and theoretical coding [31]. First, an initial set of interviews were transcribed and openly coded using atlas.ti. Open coding involves "attaching a code or label that summarizes and accounts for each piece of data" [31: 43]. In our case, this involved attaching a code to sections of the transcribed interviews or document that indicated a concern (e.g. alternatives or competing options or dilemmas that confronted Trafiklab in advancing the ecosystem) and potential actions taken in response. The first round of coding was descriptive and broad as we sought to gain an empirical understanding of the case while being open to exploring our studied phenomenon. Second, in later rounds of interviews we investigated further concerns and actions that occurred frequently in the data. These subsequent interviews explored in detail how specific actions taken were related to resolving an identified issue. The material was analyzed through "focused coding" [31: 57] in which frequently occurring codes identified in the initial open coding were used to support, synthesize and explain larger segments of data. In doing so, we focused on specific actions Trafiklab took towards resolving those concerns. To ensure we had not missed any relevant aspects, we used the initial concerns and actions identified to develop a timeframe of actions and concerns with which we held further interviews with four data providers exploring their views of those concerns. Based on further interviews, proposed minor changes were made to some concerns we had identified and their timing. Based on this, we reworded codes, merging some together. For example, codes related to standardizing data formats, upgrading APIs, data integration etc. were broadly categorized under the label "data quality".

Thirdly, once we had identified concerns that were relevant and for which Trafiklab took actions to address, we held further interviews with employees who could be knowledgeable about these identified concerns. In doing so, we described the concern in terms of the successive actions Trafiklab took. For example, Trafiklab wanted to advance certain technically-related functions of the platform by reaching out to users through informal meetings e.g. meet-ups or using newsletters. We then explored why this was necessary. For example, codes that labeled the different ways Trafiklab reached out to users and providers through Meet-ups or hackathons, and using their support forum, were grouped under the core category interactions. Through multiple iterations and discussions between the authors, we examined relationships between the different core categories, exploring trends of the changes through axial coding [31]. The outcome is the general process patterns described in figure 1, and the details of how this process evolved described in the following.

\section{Navigating Tensions from Competing Concerns}

Figure 1 below shows an inductively identified iterative process generated from our analysis in which the platform provider sought to navigate competing concerns and associated tensions in the establishment of an ecosystem. The process logic in the model is such that emergent practices among distributed actors result in tensions i.e. new inconsistencies are generated, or become salient, contradictory or disabling [4]. Tensions trigger a new platform configuration (i.e. sociotechnical structures constituted by the platform core, complementary resources, and couplings to modules and actors in the ecosystem) to materialize. The new platform configuration induces a value logic (i.e. specific composition of architectural leverage i.e. production, innovation and transaction [26]) guides interactions i.e. exchange of various kinds between actors in value creation processes based on information transactions and exposure to diverse resources. For Trafiklab, we found this pattern to repeat in multiple cycles described in phases 1-4. Below, we describe phases characterized by the identified tensions that triggered these dynamics, and how the platform provider sought to navigate them. 


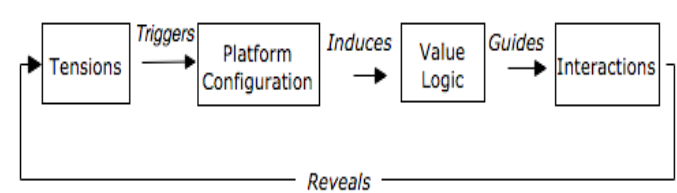

Figure 1. Platform dynamics

\subsection{Phase 1: Internal vs. External service development}

The launch of Trafiklab in September 2011 was ignited by a practical concern: the failure of major PTOs to adjust daily operational routines and services (e.g. customer support, communications, delivery of services) swiftly to changes brought about by the use of smartphones. Third-party developers resorted to scraping data from PTOs websites, in order to develop smartphone applications for users of public transport. Samtrafiken, a joint venture aimed at coordinating and strengthening public transport services owned by 38 PTOs, made initial efforts to adapt so that it could coordinate service development with third-party developers. This was unsuccessful as developers felt that Samtrafiken's desire to retain control restricted them, resulting in low take-up of these alternatives and a continued preference to scrape the PTO data. The PTOs were left with the options of either making improvements to their existing services or shutting down third-party services entirely. Shutting down other applications based on scraped data was not feasible since these were based on publicly available, open data. This was a problem for the PTOs since issues with these applications, such as incorrect use of information or low-quality programming, could result in poor or faulty information to public transport users. This triggered the need for an alternative that would address both the developers' concerns and those of the PTOs. As the manager of Trafiklab explained:

"If we did not develop this platform, third-party developers would still build applications using our data. We wanted to offer a structured process where developers could develop services that would not conflict with our own services, because we had initially experienced problems when developers-built services using scraped data." [Trafiklab Manager]

Instituting new sociotechnical design changes: Settling for the option to steer developers away from scraped data to an alternative service development model resulted in the creation of Trafiklab, a platform to coordinate the activities of third-party developers and PTOs. The initiative involved the development of a transformation layer: an architectural layer for data filtering and standardization that provided a shared resource for future actors joining Trafiklab. For Trafiklab, the architectural layer acted as an incentive to entice data providers to the platform, since APIs could be tested and exposed to third parties without them incurring the full cost of developing a proprietary layer. The transformation layer provided potential developers an alternative to scraped data as it offered a filter function that transformed and generated multiple data formats. The purpose was to provide an agile environment for developers, reducing the time and cost involved in accessing data. The idea was to align Trafiklab with the developers' "culture", distinguishing it from bureaucratic public transport, and to provide a better option than scraping data from different PTOs. As the manager explained:

"In the beginning, we use the transformation layer to develop APIs that were more suitable for developers to create services rather than data scraping." [Trafiklab Manager]

Incentivizing participation to enable value exploration: As Trafiklab attracted developers and data providers, it built on these initial users to further consolidate its user base through different incentives. For example, prizes were awarded to developers participating in innovation competitions. Through travel hacks, specific tasks were assigned to developers to explore possible services. For example, an App Contest to create apps for disabled travelers focused on addressing the needs of the deaf and blind travelers. Trafiklab partnered with other institutions in organizing travel hacks. This served as a way to attract developers and data providers, and, inform stakeholders of the potential value in public transport data. As explained by the manager:

"We have arranged travel hacks together with other institutions..... as a way to attract developers to look at new kinds of services...and to keep stakeholders informed of our work" [Trafiklab Manager]

Stimulating interactions between data providers and developers: To address user needs better and encourage enrollment on the platform, Trafiklab facilitated open interactions between data providers and developers aimed at knowledge-sharing among the initial users. Besides using its support forum, the Trafiklab Meet-ups were particularly valuable for engaging with users. Thus, a network of developers and data providers could share experiences and ideas on using Trafiklab at workshops and physical meetings. An added advantage of meet-ups was that developers interacted directly with data providers.

\subsection{Phase 2: Stability vs. Adaptability of platform resources}


As Trafiklab grew over time, it became apparent that the initial set of resources e.g. APIs available on its platform significantly limited the developers. This realization was largely related to the recognition that most application prototypes did not result in large-scale services and could not easily adapt to frequent changes associated with APIs. To remain relevant in the ecosystem, Trafiklab needed to move beyond the mere publication of data and, demonstrate the potential value, which they reckoned could further expand the user base and scope of the ecosystem.

"To make the ecosystem around Trafiklab grow, it was not enough to make an app prototype... We wanted professional apps ... Initially, we focused on providing APIs for third-parties... But this limited the options that developers could use the APIs for." [Business Strategist]

Adopting new sociotechnical resources to scale services: Realizing that it needed to expand its user base and scale of services, Trafiklab made a concerted effort to develop APIs with functionalities and formats interoperable with other data formats used across developer communities. There was a recognition that, despite the strength inherent in the position as a national hub, Samtrafiken and Trafiklab could not, and would not, benefit from imposing their own technical practices. Thus, Samtrafiken and Google started a collaboration to provide data through APIs supporting the GTFS (General Transit Feed Specification), a data format used by Google Transit for applications within public transport.

"Samtrafiken had a collaboration with Google. We provided our data for Google to publish. We launched GTFS files, the global standard format for public transport data.... it is easy for developers to when using our data." [Trafiklab Manager]

Leveraging ecosystem relationships to support value creation: As Trafiklab shifted from providing access to data to developing APIs for data formats interoperable with other formats used across developers' communities, the existing relationships with data providers became an increasingly valuable resource that they provided developers with. Trafiklab initiated a first line of support, which involved support for third-party developers in response to questions e.g. updates related to an API that required a swift response. Given Trafiklab's knowledge about the personnel working in the different PTOs, it leveraged this position to forward inquiries swiftly to appropriate people with specialized knowledge about the problems raised, in case Trafiklab did not have the competency to provide a definite answer to the developer. In doing so, Trafiklab simplified things for developers and further strengthened its position as a more agile environment responding to developers' needs.
Leaning interactions towards future changes: Given that changes in data format could, in some cases, be planned but in others be unforeseen as services scaled across other data formats, Trafiklab provided a roadmap to developers of possible directions that future services and APIs might take. Trafiklab communicated APIs updates to specific affected developers through newsletters. Although these changes could be communicated in the developer's forum, some cases involved data providers who did not use the support forum. Overall, these measures aimed to provide a sense of direction for developers and ensure interoperability of services across different data formats.

“...we keep developers updated of specific changes on APIs that will affect their work" [IT support staff]

\subsection{Phase 3: Quality vs. Quantity of services developed}

As Trafiklab attracted more developers and increased the number of different data formats for the development of services, it witnessed the challenge of controlling and ensuring the quality and variety of services. This concern affected both data providers and Trafiklab. For Trafiklab, this could jeopardize the relationship it had with providers as they could be wary that their data was being published wrongly or used by developers in unacceptable ways, which could cause problems for public transport users.

"The main risks we are facing is that customers are using services we cannot control and if these services are shut down, or not updated, it becomes tricky for us to take care of all customers dependent on the service. [PTOs] might think we are the cause" [Trafiklab Manager]

Matching sociotechnical resources with service standards: To ensure the output of services was of better quality and of an acceptable standard, developers had to sign agreements before they could start working on the APIs. In this way, Trafiklab could shut down applications considered inappropriate according to the standards, an action they rarely undertook, as developers could resort to scraping data as these data were publicly available. Samtrafiken's earlier attempts to control third-party development through restrictive measures had ended unsuccessfully. A key management system with API keys was thus used to ensure control and maintain service quality.

"developers need to sign up for APIs keys and, agree on how the data is used. We can shut down services that break those terms." [Head of IT]

Incentivizing participation and value through progressive access: Trafiklab developed a progressive 
scale for access by different developers through the key management system, a level of access that could be extended based on the adoption and usage of the applications developed. By instituting a progressive scale, Trafiklab saw this as an incentive for developers to develop better services or risk being restricted to lower levels of requests and APIs access. Such incentives were a component in Trafiklab's continuous ambition to move beyond prototype apps and reduce load in its backend systems.

"The bronze level is 10,000 requests/month, the silver is 100,000, the gold is 10-12 million... This is an incentive for developers to move to the next level and for us to control traffic in our backend systems... " [IT support]

Matching interaction and access with output of services: Beyond service agreements, control of third parties and service quality was also achieved using different levels of access to ensure access and service quality varied according to the quality of the developed applications and the usage of the services developed. Different configurations of APIs were embedded as part of the key management system. API Keys were matched to the projects of developers. This provided feedback and monitoring of the output of specific projects and usage of an applications.

\subsection{Phase 4: Resource efficiency vs. Scaling capacity of platform resources}

As Trafiklab had attained the initial objective of creating an environment for developing services, attracting about 3000 users, and having 2200 developed services, it sought to ensure that its ecosystem of developers and data providers was sustainable for a long haul both in terms of efficient utilization of resources and scaling the services developed. This was in recognition of the fact that developers benefited little financially from the initial services developed as they engage largely in hobby projects with low capacity to scale. Options were considered to enable developers to sell tickets through smartphones. Trafiklab's view was that both developers and data providers could be attracted to the platform in the long-term through network effects triggered by APIs that would enable ticket sales.

"Developers can't sell tickets...developers could create more services if they can make money from the services. The only way they can make money is to involve them in new ways of selling tickets." [Business Strategist]

The decision to enable a monetary base for Trafiklab was, however, a concern for PTOs, who wanted to keep control over this domain. The idea of ticketing was also problematic from Samtrafiken's perspective, given that it required service agreements with other stakeholders, a political process requiring resources and technical competences. For example, systems to handle validation of tickets, and a mandate from different PTOs needed to be negotiated. Apart from being unsuccessful in realizing the idea of APIs with the functionality to handle the sale of tickets, Trafiklab faced an unexpected event in its ecosystem. SL, a key data provider for Trafiklab's ecosystem, decided to move to a new proprietary platform to handle data transformation internally. Data transformation had become critical for SL's core business and it needed to ensure control and align the system with its organizational needs.

"SL bought new systems to transform APIs. Instead of being a shared resource, the new systems are in their control. The transformation layer became critical for SL's core business... [Trafiklab Manager]

Streamlining sociotechnical resources: The decision by SL to move to a proprietary platform created profound and unforeseen consequences for Trafiklab. The transformation layer had, up until now, been one of Trafiklab's key resources, but maintaining it to filter fragmented data and test new APIs became costly and its value was questioned as providers developed proprietary solutions. Thus, Samtrafiken decided to shut down the transformation layer.

"There were lots of different issues regarding the transformation of data. It was costly to maintain given the resources we had. So, we shut it down." [Business Strategist]

Refining self-identity to passively sustain ecosystem: The removal of the transformation layer resulted in a loss of control for Trafiklab as access to APIs of key actors became restricted. It became difficult for smaller actors to join Trafiklab. The layer was a gateway for transforming data and an entry point to the ecosystem for data providers to test and explore the benefits of APIs before committing.

"To have small actors joining Trafiklab now, they need to build new API systems. This is an entry barrier. Before, they could test APIs by connecting their internal APIs systems with the transformation layer. Now, they have to build the layer on their own, which is costly." [Trafiklab Manager]

SL's departure and the shutdown of the transformation layer challenged Trafiklab's identity. To some, Trafiklab was a marketplace, to others a brand, a community for developers. Evident was that Trafiklab's role and identity was less clear.

"We've had lots of question about our work. We needed to change. Now, Trafiklab is a brand, a marketplace. It's a community for developers. There are many views." [Business Strategist] 


\section{Discussions}

Most studies examining competing concerns and resulting tensions do so from the vantage point of an incumbent platform provider [3, 19]. Described as platform leaders, prior literature uniquely positions these incumbents as powerful actors in the ecosystem through the "bouncer rights" they exercise via control of strategic resources [6, 10, 13]. However, how providers navigate competing concerns and resulting tensions, in emerging platform ecosystems has received little attention.

Based on our analysis of Trafiklab, we propose a process model of the dynamics involved in this navigation process (see figure 1). The model focuses on how (a) tensions from competing concerns or inefficiencies in existing/alternative solutions trigger materialization of a (b) platform configuration. We suggest that a platform configuration (i.e. the sociotechnical structures constituted by the platform core and its couplings to modules) emerges in response to tensions based on perceived inefficiencies, appreciation of alternative solutions or divergent interests. The platform configuration suggests a value logic. (c) The value logic (i.e. specific composition of architectural leverage in production, innovation and transaction [26] may or may not have been foreseen in the design. Types of leverage are not mutually exclusive, rather different configurations enable different amount and types of the three logics. For example, in the Trafiklab case the core value logic shifted with the removal of the transformation layer, from an emphasis on innovation leverage to a transaction-oriented logic. The value logic guides (d) interactions among actors in the ecosystem. From the emergent practices and adaptive behavior arising through interactions and appropriation of the value logic, new tensions are triggered. For platform providers, it is a salient challenge to understand the types of interactions they guide users toward. The actors in the ecosystem are gradually shaping an understanding of value creating interactions through trial and error practices. During the course of such learning processes, ecosystem actors are likely to engage in practices that trigger new tensions.

Our study demonstrates that emerging platform providers have a relatively low strategic leverage to influence the trajectory of a platform and ecosystem. Given the need to legitimize and scale activities across the ecosystem, emerging platform providers align with incumbent actors whose actions expose the platform to vulnerabilities. As illustrated by the departure of key actors and removal of the transformation layer in our case, the emergent nature of order creation in complex systems [32] might lead the provider to unassumingly implement new configurations (in responses to tensions) that alters the architectural leverage and questions the platform's identity. These findings suggest that emerging platforms should adopt strategies enabling them to diversify their value logic and operate across multiple ecosystems rather than focusing on one key actor or ecosystem [18]. We identified four salient tensions, each characterizing different phases in Trafiklab's trajectory, and associated responses driving these dynamics. Below we discuss these four tensions, how they shaped Trafiklab's trajectory, and attempts to navigate them.

Consolidating and coordinating autonomous actors: As the value of platforms is partly related to the size of the user base, attracting the first actors in the ecosystem is a salient challenge. A platform provider needs to balance disparate interests so that each relevant group finds the value logic compelling enough to adopt the platform. The control vs. design options tension poses a challenge for platform providers as potential users, due to the uncertainty of the new platform can be reluctant to embrace it. Trafiklab sought to consolidate ecosystem actors by leveraging an architectural layer that provided resources aligned with developer's interests, while still providing a sense of increased control towards data providers. Trafiklab had a relatively weak bargaining position as developers could turn back to their data scraping practices if the value logic was not attractive enough. Thus, Trafiklab largely focused on aligning with developers by e.g. simplifying search and access to data, providing incentives such as prizes to developers to explore APIs. Our study suggests that while access and control provides a platform provider leverage to influence actors in the ecosystem [6], enabling value-driven lock-ins is another potential governance mechanism. Trafiklab's focus on consolidating users by designing boundary resources more compelling than the scrapping option is an example of such value-driven governance. Also, Trafiklab's boundary resources were not restricted to APIs but also included social resources to spur interactions among developers. The importance of these interactions suggests that social boundary resources requires more attention in research.

Ensuring stability vs. adaptation of platform: In the early stages of a platform, the user base provides not only resources but also feedback and ideas informing discovery of, and decisions on, strategic design options [33]. Observing actions and engaging in interactions with distinct user groups facilitate discovery. Simultaneously, innovation in the larger technological environment might generate other attractive options to scale the user base. However, an essential tenet in platform architecture is to keep the core stable and 
maintain a relatively low degree of adaptations of interfaces to minimize disturbances for peripheral modules. This challenge is not unique to emerging platforms but rather applies to all platforms carrying non-trivial degrees of digital components that must be adapted relatively frequently due to the rapid pace of external innovation. However, users of emerging platforms are likely to devote less resources to track changes in interfaces and adapting their modules. At Trafiklab, the response from interactions within and across actors provided feedback on potential advantages and disadvantages of certain configurations. Adaptation to achieve scaling of the platform's services was largely based on the developers' perspective but with the ultimate aim of generating end-user services that could scale and prove the value of the ecosystem to all actors. To mitigate drawbacks of frequent technical adaptation, Trafiklab devoted substantial resources to communicate technical changes to key actors. In line with prior studies, our case suggests that communicating planned changes can incentivize developers to update and refine services, thus building the user base of a platform [34].

Managing quality vs. quantity of services developed: Digital platforms are valuable because they create opportunities to engage distributed actors. Striking a balance between quality of the services while not overly constraining access and freedom to variety, is a key tension that platform providers face. For Trafiklab, tracking the quality of services generated by users was a key measure to handle this tension. The platform resources were configured to provide incentives through different degree of access to the platform for developers based on the services they created. Since the value of Trafiklab's "bouncer right" was still relatively weak, these incentives mainly came in terms of carrots rather than sticks.

Managing resource efficiency vs. Scale capacity: While potential platform providers and ecosystem actors can initially share a common goal driven by a common vulnerability, efficient utilization of resources in the long-term to scale a platform as it evolves remains challenging. Because of the dependence on key actors for resource provision, platform providers in emerging ecosystems might be attracted, to resolve this tension by adapting socio-technical resources in ways that comprise their interest. In our case, the platform provider scaled down sociotechnical resources as key actors departed from the ecosystem, resulting in a platform reconfiguration that increased the adoption cost for other actors to join the ecosystem. The outcome was a shift of the value logic from an emphasis on innovation through shared resource integration, to a transaction-oriented logic where facilitation of knowledge exchange became dominant.
This had implications on the platform's ability to actively govern the ecosystem not only because it effectively hindered supply-side growth of the ecosystem, but also came to challenge the platform's identity. Because the identity and use patterns in early phases of a technology is highly open for interpretation by users [35], relatively minor changes in design might have significant impact on their trajectory.

\section{Conclusion and Future Work}

Our research at Trafiklab shows that a platform's configuration has significant implication for the value logic presented to prospective users, the interactions facilitated and the distribution of value in the ecosystem. Platforms that have not reached a critical mass of users remain weak, as the costs associated with losing access to the ecosystem for actors that choose to fully or partly abandon the platform are relatively low. The weak bargaining power suggests that emerging platforms become vulnerable in regard to tensions perceived by key users, which might result in these users changing their relationships with, and the use of, the platform. The dependency on key users might also lead platform providers to redesign a platform in ways not aligned with their long-term strategy. When evaluating such options, providers need to consider how changes in platform configurations can lead to significant change patterns. Our study is situated in the domain of public/private collaborations, focusing on a platform engaged in the provision of open APIs and public data. Thus, regulations and competing concerns might be particularly salient in this context. The importance of these contextual specifics requires further attention.

\section{References}

[1] A. Tiwana, Platform ecosystems: aligning architecture, governance, and strategy. Burlington, MA: Morgan Kaufmann, 2014.

[2] M. de Reuver, C. Sørensen, and R. C. Basole, "The digital platform: a research agenda," Journal of Information Technology, 2017, pp. 1-12.

[3] A. Ghazawneh and O. Henfridsson, "Balancing platform control and external contribution in third-party development: the boundary resources model," Information Systems Journal, 2013, Vol. 23, No. 2, pp. 173-192.

[4] J. Wareham, P. B. Fox, and J. L. Cano Giner, "Technology ecosystem governance," Organization Science, 2014, Vol. 25, No. 4, pp. 1195-1215.

[5] B. Eaton, S. Elaluf-Calderwood, C. Sorensen, and Y. Yoo, "Distributed tuning of boundary resources: the case of Apple's iOS service system," MIS Quarterly, 2015, Vol. 39, No. 1, pp. 217-243. 
[6] A. Gawer and R. Henderson, "Platform owner entry and innovation in complementary markets: Evidence from Intel," Journal of Economics \& Management Strategy, 2007, Vol. 16, No. 1, pp. 1-34.

[7] R. Lindgren, O. Eriksson, and K. Lyytinen, "Managing identity tensions during mobile ecosystem evolution," Journal of Information Technology, 2015, Vol. 30, No. 3, pp. 229-244.

[8] A. Gawer, "Bridging differing perspectives on technological platforms: Toward an integrative framework," Research Policy, 2014, Vol. 43, No. 7, pp. 1239-1249.

[9] M. Schreieck and M. Wiesche, "How established companies leverage IT platforms for value co-creationinsights from banking," presented at the 25th European Conference on Information Systems (ECIS), Guimarães, Portugal, 2017.

[10] K. J. Boudreau, "Platform Boundary Choices \& Governance: Opening-Up While Still Coordinating and Orchestrating," in Entrepreneurship, Innovation and Platforms, vol. 37, A. G. J. Furman, B.S. Silverman, S. Stern, Ed.: Emerald Publishing Limited, 2017, pp. 227-297.

[11] L. J. Strahilevitz, "Information asymmetries and the rights to exclude," Michigan Law Review, 2006, Vol. 104, No. 8, pp. 1835-1898.

[12] F. M. Santos and K. M. Eisenhardt, "Organizational boundaries and theories of organization," Organization science, 2005, Vol. 16, No. 5, pp. 491-508.

[13] K. Boudreau and A. Hagiu, "Platform rules: Multi-sided platforms as regulators," in Platforms, Markets and Innovation, A. Gawer, Ed. London: Edward Elgar, 2008, pp. 163-191.

[14] A. Hagiu, "Pricing and commitment by two-sided platforms," The RAND Journal of Economics, 2006, Vol. 37, No. 3, pp. 720-737.

[15] T. Eisenmann, G. Parker, and M. W. Van Alstyne, "Strategies for two-sided markets," Harvard business review, 2006, Vol. 84, No. 10, p. 92.

[16] D. Evans, "How catalysts ignite: The economics of platform-based start-ups," In Platforms, Markets and Innovations, A. Gawer, Ed.: Edward Elgar Publishing, London, 2009, pp. 99-128.

[17] J. Claussen, T. Kretschmer, and P. Mayrhofer, "The effects of rewarding user engagement: the case of facebook apps," Information Systems Research, 2013, Vol. 24, No. 1, pp. $186-200$.

[18] L. Selander, O. Henfridsson, and F. Svahn, "Capability search and redeem across digital ecosystems," Journal of Information Technology, 2013, Vol. 28, No. 3, pp. 183-197.

[19] A. Gawer and M. A. Cusumano, "How companies become platform leaders," MIT Sloan Management Review, 2008, Vol. 49, No. 2, p. 28

[20] K. Lyytinen, Y. Yoo, and R. J. Boland Jr, "Digital product innovation within four classes of innovation networks," Information Systems Journal, 2016, Vol. 26, No. 1, pp. 47-75.

[21] J. L. Zittrain, "The generative internet," Harvard Law Review, 2006, pp. 1974-2040.

[22] Y. Yoo, R. J. Boland, K. Lyytinen, and A. Majchrzak, "Organizing for Innovation in the Digitized World," Organization Science, 2012, Vol. 23, No. 5, pp. 1398-1408.

[23] T. Donaldson and L. E. Preston, "The stakeholder theory of the corporation: Concepts, evidence, and implications," Academy of management Review, 1995, Vol. 20, No. 1, pp. 65-91.

[24] W. K. Smith and M. W. Lewis, "Toward a theory of paradox: A dynamic equilibrium model of organizing," Academy of Management Review, 2011, Vol. 36, No. 2, pp. 381-403.

[25] B. Caillaud and B. Jullien, "Chicken \& egg: Competition among intermediation service providers," $R A N D$ journal of Economics, 2003, Vol. 32, No. 2, pp. 309-328.

[26] L. D. Thomas, E. Autio, and D. M. Gann, "Architectural leverage: putting platforms in context," The Academy of Management Perspectives, 2014, Vol. 28, No. 2, pp. 198219.

[27] S. Nambisan, K. Lyytinen, A. Majchrzak, and M. Song, "Digital Innovation Management: Reinventing Innovation Management Research In a Digital World," MIS Quarterly, 2017, Vol. 41, No. 1, pp. 223-238.

[28] A. Afuah and C. L. Tucci, "Crowdsourcing as a solution to distant search," Academy of Management Review, 2012, Vol. 37, No. 3, pp. 355-375.

[29] W. M. Cohen and D. A. Levinthal, "Absorptive capacity: a new perspective on learning and innovation," Administrative Science Quarterly, 1990, Vol. 35, No. 1, pp. 128-152.

[30] R. F. Lusch and S. Nambisan, "Service innovation: A service-dominant logic perspective," Mis Quarterly, 2015, Vol. 39, No. 1, pp. 155-176.

[31] K. Charmaz, Constructing grounded theory: A practical guide through qualitative analysis. London: Sage Publications Ltd, 2006.

[32] J. H. Holland, Emergence: From chaos to order. Perseus Publishing, Boulder CO, 1998.

[33] J. Sandberg, L. Mathiassen, and N. Napier, "Digital Options Theory for IT Capability Investment," Journal of the Association for Information Systems, 2014, Vol. 15, No. 7, pp. 422-453.

[34] A. Gawer and M. A. Cusumano, "Industry platforms and ecosystem innovation," Journal of Product Innovation Management, 2014, Vol. 31, No. 3, pp. 417-433.

[35] M. Tripsas, "Technology, identity, and inertia through the lens of "The Digital Photography Company"," Organization science, 2009, Vol. 20, No. 2, pp. 441-460. 